recognised problem which psychiatrists will have to face in future work.

Psychiatrists are also examining ways of avoiding hospitalisation by offering greater support at home. In Camberwell, a deprived inner city area, a research team randomly allocates psychotic patients seen in the emergency room to a community based daily living programme (DLP) as an alternative to standard hospital care. The team aims to maximise functioning in the community by offering greatly increased support to patients and their families at times of particular need, while having key-workers to improve continuity of care and co-ordination of services. Over the last 18 months, although three quarters of the DLP patients have had subsequent hospital admissions, these have generally been brief, half lasting less than three days. Patients' families appreciate the support offered and with their involvement, patients are more compliant with treatment, so reducing the need for compulsory admission. In particular, the researchers have noticed better compliance with treatment among ethnic minority patients and their families.

American psychiatrists have demonstrated the need to go on providing a high level of support even after apparent full recovery. Patients on a one year training in community living (TCL) programme did well during active management. However, at the end of the one year trial period when standard follow-up only was available, individuals were unable to maintain the gains they had made in social functioning leading to improved satisfaction with life. Instead they became more symptomatic with increased hospitalisation. Psychiatrists have also tried to develop preventive measures by opening drop-in clinics. These tap a new patient group, the 'worried well'. In order to reach more acutely disturbed people in the community, a more active approach to crisis intervention is generally needed. Community team members routinely make emergency home assessments, offering appropriate treatment so as to avoid unnecessary hospital admission.

\title{
Miscellany
}

\section{Queen's Birthday Honours List}

Professor James Griffith Edwards, Institute of Psychiatry, has been awarded the CBE and Dr Hector Fowlie, formerly Vice Chairman, Mental Welfare Commission for Scotland, has been awarded the OBE.

\section{New appointment}

MIND, the mental health charity, has appointed Ros Hepplewhite as its new National Director. She was previously Director of Corporate Development with Brighton Health Authority and has also held management responsibility for mental health services in a teaching hospital, an inner city area and as a Unit General Manager in Brighton.

\section{Palestinian Mental Health Association}

Amaan, the Palestinian Mental Health Association, has been set up recently to deal with the psychological and emotional problems that affect the people of Palestine. Any donations of books and research papers (published since 1985) will be very welcome, as will financial contributions. Further particulars and donations to Sheena Dunbar, Amaan, P.O. Box 2516, Cairo 11361, Egypt or contact: Dr Suman Fernando, Department of Psychiatry, Chase Farm Hospital, Enfield, Middlesex.

\footnotetext{
"What you do in this world is a matter of no consequence... the question is, what can you make people believe that you have done."
}

Sir Arthur Conan DOYLe 